\title{
3D multi-spectral image-guided Near-Infrared spectroscopy using the boundary element method
}

\author{
S. Srinivasan, B. W. Pogue \& K. D. Paulsen \\ Thayer School of Engineering, Dartmouth College, Hanover, NH-03755, \\ USA
}

\begin{abstract}
Image guided (IG) Near-Infrared spectroscopy (NIRS) has the ability to provide high-resolution metabolic and vascular characterization of tissue, with clinical applications in the diagnosis of breast cancer. This method is specific to multimodality imaging where tissue boundaries obtained from alternate modalities such as MRI/CT, are used for NIRS recovery. IG-NIRS is severely limited in 3D by challenges such as volumetric meshing of arbitrary anatomical shapes and the computational burden encountered by existing models that use the finite element method (FEM). We present an efficient and feasible alternative to the FEM using the boundary element method (BEM). The main advantage is the use of surface discretization, which is reliable and more easily generated than volume grids in 3D and enables automation for large numbers of clinical data-sets. The BEM has been implemented for the diffusion equation to model light propagation in tissue. Image reconstruction based on the BEM has been tested in a multi-threading environment using four processors, which provide $60 \%$ improvement in computational time compared to a single processor. Spectral priors have been implemented in this framework and applied to a three-region problem with a mean error of $6 \%$ in recovery of NIRS parameters.
\end{abstract}

Keywords: NIR spectroscopy, image guided, boundary element method.

\section{Introduction}

NIR optical imaging has the ability to provide non-invasive functional characterization of tissue relating to its metabolic and vascular status [1]. This yields unique intrinsic information regarding the composition of tissue, 
not available from conventional imaging modalities such as MRI, x-rays or ultrasound, which depend on structural features for contrast. NIR spectroscopy and tomography have been used to study breast tissue for the diagnosis of breast cancer [2], monitoring neoadjuvant chemotherapy [3] and assessing risk of cancer through screening [4]; as well as in other areas such as the study of brain physiology [5], muscle [6] etc. Our particular interest is in exploring the use of NIR imaging to improve the specificity of breast cancer diagnosis [7].

The high scattering in tissues like the breast allow penetration of light up to a dozen centimeters, crucial to deep-tissue imaging; however this same scattering also limits the spatial resolution of NIR imaging to millimeter scale. Over the past decade, research has explored the synergy of hybrid multi-modality imaging by combining modalities such as MRI, ultrasound or x-ray with NIR to increase the spatial resolution and improve sensitivity and specificity of breast cancer diagnosis [8-10]. At Dartmouth, a hybrid MRI-NIR system has been developed for this purpose [8].

The image reconstruction algorithms developed for hybrid systems have studied various intelligent ways of incorporating the anatomical information from MRI, x-ray or CT, into the ill-posed NIR image recovery process $[11,12]$. The most intuitive and accurate results have been obtained using the 'hard priors' algorithm which assumes the domain to contain piece-wise constant regions whose exact boundaries are obtained from the alternate modality such as MRI [11, 13]. This produces the best global estimates of NIR vascular and structural parameters such as total hemoglobin, oxygen saturation, water, scatterer size and density. Currently, FEM techniques are utilized for this purposes which have severe drawbacks in 3D IG-NIR reconstructions namely: (1) need for volume discretization of arbitrary breast shapes which is a complex procedure and does not lend easily to automation of large clinical data sets, and (2) requirement of large meshes for accuracy ( $>30000$ nodes) of the FEM method, though reconstructing for only few parameters ( $10-15$ values): this has implications in computational time and efficiency. To overcome these issues, we have developed BEM for IG-NIRS reconstructions.

BEM is an attractive alternative numerical method requiring only surface discretization, which are faster and more reliably produced as compared to volume meshes. BEM uses the assumption that the imaging domain contains known number of piece-wise constant regions whose boundaries are obtained from MRI, which is the same as hard priors. The BEM is uniquely suited for IGNIRS, since the breast typically consists of limited tissue types such as adipose, fibroglandular tissues and the lesion (malignant and/or benign), and the exact knowledge of these tissue layers can be obtained from MRI. Towards this end, we have developed a forward model to the diffusion equation based on the BEM [14], which solves for fluence given a distribution of optical properties in the tissue domain. Image reconstruction involves solving the inverse problem of estimating optical properties of the tissue types given a set of measurements of fluence in the domain; this has been implemented using the Newton's method. Addition of spectral constraints to reconstruct for wavelength-independent 
parameters have significant impact on the accuracy of total hemoglobin, oxygen saturation, water and scattering $[7,15]$; and have been implemented in the BEM framework. We present application of this 3D BEM-based multi-spectral IGNIRS in simulations and discuss future applications in clinical setting.

\section{Methods}

The forward model is represented by the diffusion equation involves obtaining the light flux outgoing from different surfaces, using known optical properties for the interior of these regions. The diffusion approximation to the radiative transport equation assumes that the interior photon irradiance is highly scattered and nearly uniform in all directions, and therefore, its angular distribution is effectively described by the single isotropic fluence parameter, $\Phi$ [16], and is given by:

$$
-\nabla . D(r) \nabla \Phi(r, \omega)+\left(\mu_{a}(r)+\frac{i \omega}{c}\right) \Phi(r, \omega)=q_{0}(r, \omega)
$$

When tissue is assumed to consist of piece-wise constant homogeneous regions, the diffusion coefficient, $D(r)$ is constant in each zone so that the diffusion equation can be expressed in the form of a modified Helmholtz equation,

$$
\nabla . D_{l} \nabla \Phi-k_{l}^{2} \Phi=-q_{0}(r, \omega)
$$

where $k_{l}$ is the wave number, constant in sub-domain $l[17,18]$, and for a particular frequency $\omega, k_{l}^{2}=\left(\mu_{a}(r)+\frac{i \omega}{c}\right)$. The fundamental Green's function solution is readily available for this equation and can be used to derive the BEM formulation[19] given as:

$$
c_{i} \Phi_{i}+\oint D_{l} \frac{\partial G_{i}}{\partial n} \Phi-\oint D_{l} \frac{\partial \Phi}{\partial n} G_{i}=\left\langle q_{0}, G_{i}\right\rangle
$$

where Gi is the Green's function for the modified Helmholtz equation given in 3D as:

$G_{i}\left(r, r_{i}\right)=\frac{\exp \left(\frac{-k_{l}\left|r-r_{i}\right|}{\sqrt{D_{l}}}\right)}{4 \pi D_{l}\left|r-r_{i}\right|} ; \quad c_{i}=\left\{\frac{\Omega}{4 \pi}\right.$ and $\Omega$ is the solid angle enclosed by the boundary at node $i$ [19] and $\oint$ is the integral over the boundary. Discretizing equation 2 through the linear basis function $\psi$ and reformulating the equation in matrix form results in:

$$
[A]\left\{\Phi_{i}\right\}-[B]\left\{D_{l} \frac{\partial \Phi_{i}}{\partial n}\right\}=\left\{Q_{i}\right\}
$$

where: $A_{i, j}=c_{i} \delta_{i j}+\oint D_{l} \frac{\partial G_{i}}{\partial n} \psi_{j} d s, B_{i j}=\oint G_{i} \psi_{j} d s$ and $Q_{i}=\left\langle q_{0}, G_{i}\right\rangle$. 
A point source representation was implemented for our purpose. A type III boundary condition on the outer boundary was used given by:

$$
\Phi_{a I}+\left.\frac{D_{I}}{\alpha} \frac{\partial \Phi}{\partial n}\right|_{a I}=0
$$

The method can be suitably extended to multiple regions by implementing continuity conditions across inner boundaries. Further details can be found elsewhere [14]. The forward model has been implemented in matlab using mex files in fortran for building the matrices and tested in simulations. Results [14] from application in two-region problems indicated that BEM was more accurate than FEM at a lower resolution; and showed 44-72\% improvement in computational time over FEM. The forward model was also demonstrated on a patient-specific mesh generated from the MRI of a mammographically normal subject [20].

Image reconstruction of optical properties was implemented based on this forward model to the diffusion equation. A modified iterative Newton's method was used for the reconstruction procedure where the least squares norm of the difference between measured data (from the NIR system) and the numerical model data was minimized [21]. A perturbation approach was used to calculate the sensitivity of the boundary measurements to change in optical properties. Regularization was used to counter the ill-conditioned nature of the problem [22] and a stopping criterion of less than $0.5 \%$ change in the error between successive iterations was applied. All reconstructions in this work were run in a multithreading environment on a Beowulf cluster using four processors.

\section{Results}

BEM-based recovery of optical properties was demonstrated previously in an experimental setting and using patient-specific mesh [14]. Recently, we have implemented the BEM to run in a multi-threaded environment using Matlab Distributive computing toolbox. In order to compare the speed-up in computational time, simulated data was generated on the imaging domain shown in Figure 1(a). The domain was described using a surface grid containing 4126 nodes generated using Netgen [23]. The measurement geometry comprised of 16 sources with 15 detectors per source ( $=240$ measurements) placed in a ring around the central plane of the domain. 1\% random Gaussian noise was added to these measurements and used to reconstruct for optical properties using a coarser surface mesh containing 2780 nodes. The recovered values for absorption and reduced scattering coefficients are shown in Figures 1(b) and (c). The recovered absorption in the inclusion was accurate within $89 \%$ and scattering was accurate within $99 \%$. The time of computation for the reconstruction using a single processor versus four processors is shown in Figure 1(d). The use of four processors provided an improvement of $59 \%$ in computational time. In all the following work, we have utilized four processors for BEM parallel computing.

Spectral priors: The known spectral signatures of the primary absorbers in tissue (see Figure 2(a)) can be embedded into the reconstruction to use multi- 


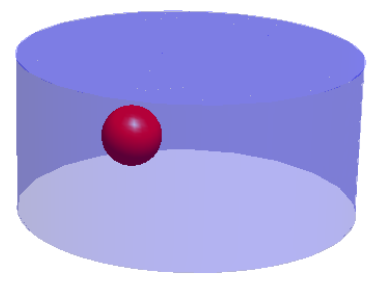

(a)

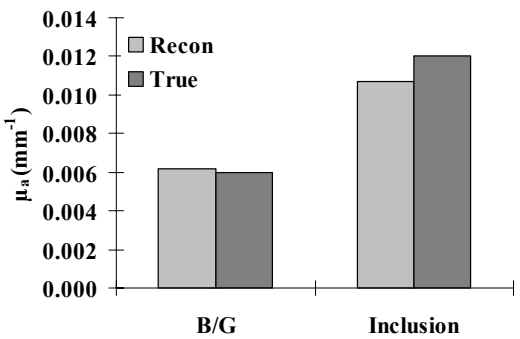

(b)

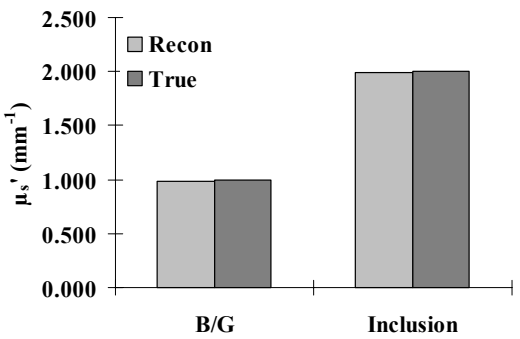

(c)

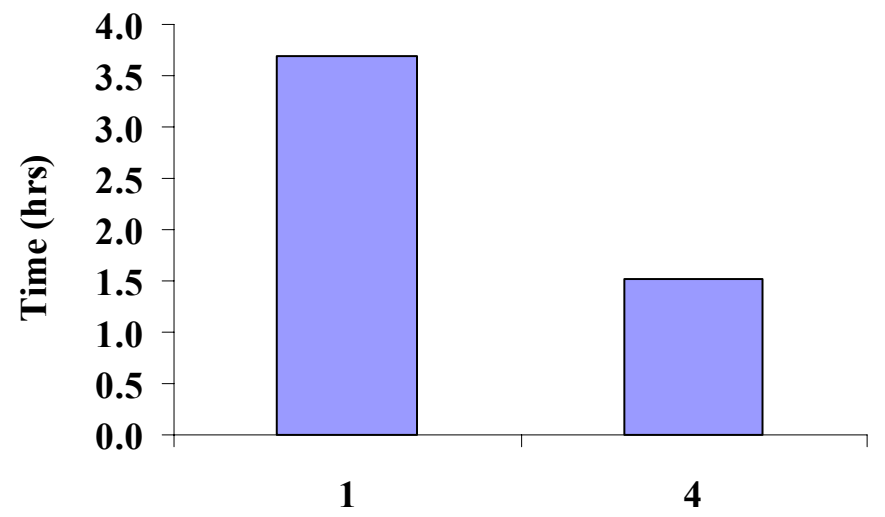

(d)

\section{Number of Processors}

Figure 1: (a) Test imaging domain used to create a surface mesh for generating forward data. The data with $1 \%$ noise was reconstructed using a coarser surface grid and recovered values are shown for absorption coefficient (b) and reduced scattering coefficient (c). (d) The differences in computational time using a single versus four processors in a multi-threaded environment are shown. 
wavelength measurements for direct recovery of chromophore concentrations (such as oxy and deoxyhemoglobin and water) and scattering parameters. This has been shown to improve the recovery of these functional parameters in the FEM framework [15] and we have implemented them in the BEM framework here. To test 3-D spectral BEM reconstruction, measurements at six NIR wavelengths were generated for a three-region test domain (shown in Figure 2(b)) with appropriate concentrations[7] to mimic the adipose, fibroglandular and tumor regions. $1 \%$ random Gaussian noise was added to these measurements and used to reconstruct for the chromophore concentrations and scattering. The recovered values are shown in Figure 3(a) and (b). The computational time was 2.15 hrs using 4 processors. The mean error in the reconstructed values over all parameters was calculated to be $0.2 \%$ in region $1,4 \%$ in region 2 and $13 \%$ in region 3. This shows the feasibility of using BEM for IG-NIRS of multi-layered domains in 3-D.

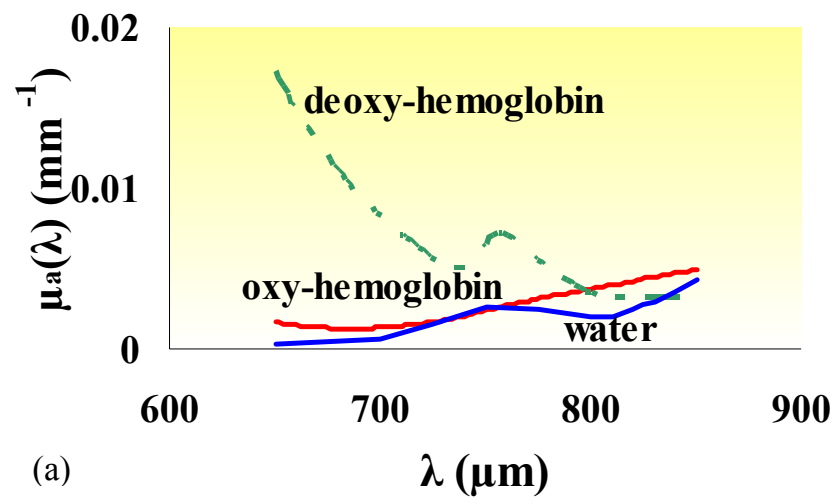

(b)

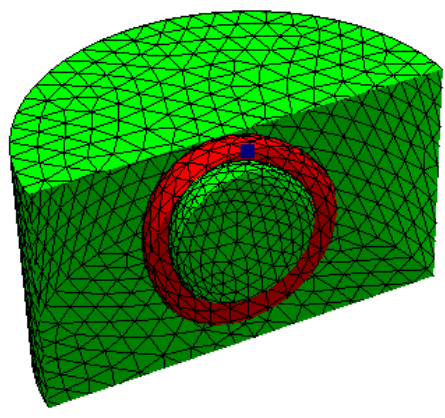

Figure 2: (a) Known spectral signatures of oxyhemoglobin, deoxyhemoglobin and water obtained from literature, can be incorporated into image reconstruction. (b) Cross-section of surface grid generated for a three-region domain, to model the multiple layers of adipose, fibroglandular and tumor layers in a breast. Appropriate concentrations were used to generate forward data. 

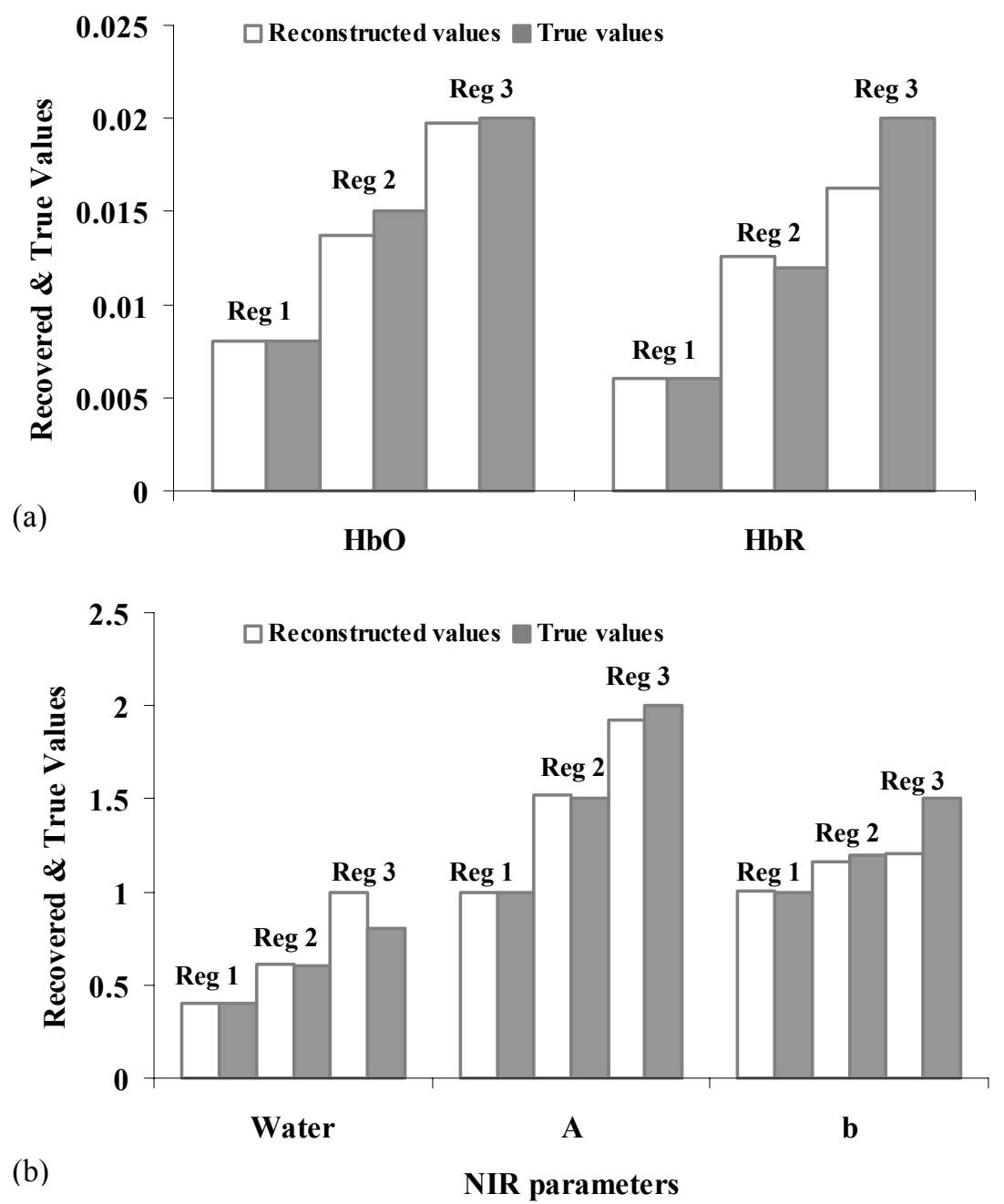

Figure 3: (a) Recovered values for oxyhemoglobin ( $\mathrm{HbO}$ ) and deoxyhemoglobin (HbR) in units of milli-molar using measurements from a three-region domain are shown. (b) Recovered values for water fraction and scattering parameters A and $\mathrm{b}$ derived from the empirical relationship $\mu_{s}^{\prime}(\lambda)=A \lambda^{-b}$.

\section{Conclusions}

A BEM approach for 3-D multi-spectral IG-NIRS has been developed and provides an efficient technique for computing in 3-D using only surface discretization. Image reconstruction using BEM was run in a multi-threaded 
environment using 4 processors and showed $\sim 60 \%$ improvement in computational time as compared to use of a single processor. We are currently limited by the number of processors and will extend it to 8 processors, planned in the future. Spectral priors were incorporated in this framework and used to reconstruct directly for chromophore concentrations and scattering in a multilayered media; results were accurate with overall mean error of $6 \%$. This method will be used to reconstruct for 3-D tissue IG-NIR spectroscopic measures using clinical in-vivo measurements at multi-wavelengths obtained from hybrid MRNIR system based at Dartmouth.

\section{References}

[1] Jobsis, F.F., Non-invasive, infra-red monitoring of cerebral and myocardial oxygen sufficiency and circulatory parameters. Science, 1977. 198: p. 1264-1267.

[2] Pogue, B.W., S.P. Poplack, T.O. McBride, W.A. Wells, K.S. Osterman, U.L. Osterberg, and K.D. Paulsen, Quantitative Hemoglobin Tomography with Diffuse Near-Infrared Spectroscopy: Pilot Results in the Breast. Radiology, 2001. 218(1): p. 261-6.

[3] Jakubowski, D.B., A.E. Cerussi, F. Bevilacqua, N. Shah, D. Hsiang, J. Butler, and B.J. Tromberg, Monitoring neoadjuvant chemotherapy in breast cancer using quantitative diffuse optical spectroscopy: a case study. $J$ Biomed Opt, 2004. 9(1): p. 230-8.

[4] Simick, M.K., R. Jong, B. Wilson, and L. Lilge, Non-ionizing near-infrared radiation transillumination spectroscopy for breast tissue density and assessment of breast cancer risk. Journal of Biomed Opt, 2004. 9(4): p. 794-803.

[5] Strangman, G., D.A. Boas, and J.P. Sutton, Non-invasive neuroimaging using near-infrared light. Biol. Psychiatry, 2002. 52: p. 679-693.

[6] Gratton, E., S. Fantini, M.A. Franceschini, G. Gratton, and M. Fabiani, Measurements of scattering and absorption changes in muscle and brain. Phil. Trans. R. Soc. Lond. B, 1997. 352: p. 727-735.

[7] Srinivasan, S., B.W. Pogue, B. Brooksby, S. Jiang, H. Dehghani, C. Kogel, W.A. Wells, S.P. Poplack, and K.D. Paulsen, Near-infrared characterization of breast tumors in-vivo using spectrally-constrained reconstruction. Technology in Cancer Research and Treatment, 2005. 4(5): p. 513-526.

[8] Carpenter, C., B.W. Pogue, S. Jiang, H. Dehghani, X. Wang, K.D. Paulsen, W.A. Wells, J. Forero, C. Kogel, J. Weaver, S.P. Poplack, and P.A. Kaufman, Image-guided optical spectroscopy provides molecular-specific information in vivo: MRI-guided spectroscopy of breast cancer hemoglobin, water \& scatterer Size. Optics Letters, 2007. 32(8): p. 933935 .

[9] Zhang, Q., T.J. Brukilacchio, A. Li, J.J. Stott, T. Chaves, E. Hillman, T. Wu, M. Chorlton, E. Rafferty, R.H. Moore, D.B. Kopans, and D.A. Boas, Coregistered tomographic x-ray and optical breast imaging: initial results. $J$ Biomed Opt, 2005. 10(2): p. 024033-0240339. 
[10] Zhu, Q., E. Conant, and B. Chance, Optical imaging as an adjunct to sonograph in differentiating benign from malignant breast lesions. Journal of Biomedical Optics, 2000. 5(2): p. 229-36.

[11] Brooksby, B., Dehghani, H., Pogue, B. W., Paulsen, K. D., Near infrared (NIR) tomography breast image reconstruction with apriori structural information from MRI: algorithm development for reconstructing heterogeneities. IEEE Journal of Selected Topics in Quantum Electronics on Lasers in Medicine and Biology, 2003. 9: p. 199-209.

[12] Boverman, G., E.L. Miller, A. Li, Q. Zhang, T. Chaves, D.H. Brooks, and D. Boas, Quantitative spectroscopic diffuse optical tomography of the breast guided by imperfect a priori structural information. Phys Med Biol, 2005. 50: p. 3941-3956.

[13] Dehghani, H., B.W. Pogue, J. Shudong, B. Brooksby, and K.D. Paulsen, Three-dimensional optical-tomography: resolution in small-object imaging. Applied Optics, 2003. 42(16): p. 3117-3128.

[14] Srinivasan, S., B.W. Pogue, C. Carpenter, P.K. Yalavarthy, and K.D. Paulsen, A boundary element approach for image-guided near-infrared absorption and scatter estimation. Medical Physics, 2007. 34(11): p. 454557.

[15] Srinivasan, S., Pogue, B. W., Jiang, S., Dehghani, H. and Paulsen, K. D., Spectrally constrained chromophore and scattering NIR tomography provides quantitative and robust reconstruction. Applied Optics, 2005. 44(10): p. 1858-69.

[16] Ishimaru, A., Wave propagation and scattering in random media. Vol. 1. 1978: Academic Press, Inc., New York.

[17] Fedele, F., M.J. Eppstein, J.P. Laible, A. Godavarty, and E.M. SevickMuraca, Fluorescence photon migration by the boundary element method. Journal of computational physics, 2005. 210: p. 109-132.

[18] Sikora, J., A. Zacharopoulos, A. Douiri, M. Schweiger, L. Horesh, S.R. Arridge, and J. Ripoll, Diffuse photon propagation in multilayered geometries. Phys Med Biol, 2006.

[19] Brebbia, C.A. and J. Dominguez, Boundary elements: an introductory course. II Edition, 1992: Computational Mechanics Publications \& McGraw-Hill Book Company.

[20] Srinivasan, S., C. Carpenter, B.W. Pogue, and K.D. Paulsen, 3D Imageguided absorption and scatter tomography using boundary element method. Biomedical Optics, OSA Technical Digest, 2008: p. BSuE36.

[21] Paulsen, K.D., and Jiang, H., Spatially varying optical property reconstruction using a finite element diffusion equation approximation. Med. Phys., 1995. 22(6): p. 691-701.

[22] Marquardt, D.W., An algorithm for least squares estimation of nonlinear parameters. J. Soc. Ind. Appl. Math., 1963. 11: p. 431-441.

[23] Schöberl, J., NETGEN - An advancing front 2D/3D-mesh generator based on abstract rules. 